\title{
Modelling Online Gaming Metacognitions: The Role of Time Spent Gaming in Predicting Problematic Internet Use
}

\author{
Gabriele Caselli $^{1,2,3} \cdot$ Claudia Marino $^{1,4} \cdot$ Marcantonio M. Spada $^{1}$ (D)
}

Published online: 13 July 2020

(C) The Author(s) 2020

\begin{abstract}
In recent years there have been growing concerns about problematic Internet use (PIU) as potential mental health problem. Among the many activities available on the Internet, the time spent gaming appears one of the most frequent risk factors in developing PIU. The aim of the current study was to model the relationship between negative affect, metacognitions about online gaming, frequency of online gaming and PIU. A total of 326 Italian gamers (mean age $=27$ years, $S D=5.65$ years; $93.3 \%$ males) participated in the study. The pattern of relationships specified by the theoretical model was examined through path analysis. Results showed that negative affect was directly associated with all other variables. Specifically, positive, strong and direct associations were found between negative affect and both positive and negative metacognitions about online gaming. Moreover, negative metacognitions about online gaming were strongly linked to PIU. Overall, the theoretical model was supported showing that metacognitions about online gaming may play a role in the association between time spent on online gaming to a broader pattern of PIU. Results are discussed within the context of the metacognitive model of psychopathology and clinical implications based on this model are outlined.
\end{abstract}

Keywords Online gaming · Metacognitions about online gaming $\cdot$ Negative affect . Problematic Internet use · Weekly online gaming hours

Marcantonio M. Spada

spadam@1sbu.ac.uk

1 Division of Psychology, School of Applied Sciences, London South Bank University, London, UK

2 Studi Cognitivi, Milan, Italy

3 Sigmund Freud University, Milan, Italy

4 Department of Developmental and Social Psychology, University of Padova, Padua, Italy 


\section{Introduction}

Problematic Internet use (PIU; e.g. Young 1998; Spada 2014) has been defined as the unregulated use of the Internet which gives rise to psychological, social, school and/or work impairments in a users' life (e.g. Beard and Wolf 2001; Moreno et al. 2019). PIU can be characterized by internet-related activities which: (1) last more than intended, leading to neglect relevant everyday tasks; (2) negatively impact on interpersonal relationships and work performance; and (3) lead to withdrawal symptoms once the access to the Internet is prevented $(\mathrm{Ng}$ and Wiemer-Hastings 2005; Cole and Griffiths 2007; Peters and Malesky 2008). PIU features share similarities to those belonging to the behavioural addictions providing credence to the assumption that PIU may be conceptualised as a coping strategy to escape/regulate or relieve negative cognition and affect (Billieux et al. 2015; Spada et al. 2008).

Research evidence suggests that PIU affects a large number of people with incidence rates ranging between 14 and 55\% (Laconi et al. 2018). It has been showed that PIU is related to time spent online, leading to a series of more severe negative consequences which include an increase in negative emotions, higher levels of self-regulation failures, and loss of relationships, career and school opportunities (Laconi et al. 2018).

PIU can be the result of multiple online activities which are not pathological per se, including information seeking, social networking, online shopping, gambling, gaming and many others. The amount of time spent in a wide range of online activities is found to be associated with PIU with online gaming showing the strongest association (Van Rooij et al. 2010). Moreover, changes in online gaming were found to be most strongly associated with changes in compulsive Internet use over time (Van Rooij et al. 2010). Concerns regarding the emergence of Internet Gaming Disorder (IGD) has been growing to the extent that the fifth edition of the Diagnostic and Statistical Manual of Mental Disorders (DSM-5, APA 2013) has presented IGD as a condition on which future research is encouraged. More recently, the World Health Organization (2018) included gaming disorder in the 11th Revision of the International Classification of Diseases (ICD-11) as a disorder aligned to addictive behaviours. The ICD-11 defined gaming disorder as a persistent and recurrent participation in digital- and video-gaming (both online and offline) which is characterized by: (1) impairment in controlling gaming behaviours, including time spent gaming and duration; (2) increasing salience of gaming to the extent to which it is preferred to other relevant activities; and (3) significant impairment in daily life (family, school, and work) and health as well as continuation of gaming despite negative consequences. In order to establish a diagnosis, these symptoms should be present for at least 12 months. However, since only a small minority of gamers suffer with IGD, the WHO suggested that monitoring the amount of time gamers spend gaming is particularly important as the difficulties in regulating such time might result in developing a problematic pattern of (Internet-related) gaming behaviour.

In view of the above, a key question emerges: How do online activities like gaming become so perseverative and poorly regulated as to turn Internet use into 
such a detrimental problem? A potential answer is provided by a metacognitive understanding of the motivational and self-regulation mechanisms involved in the frequency of online gaming and its relationship with PIU.

Metacognition can be defined as any stable knowledge about one's own cognitive system and strategies that may have an impact on the regulation of cognitions, the awareness of the current state of cognitions, and the appraisal of the meaning of thoughts and memories (Wells and Matthews 1996). Knowledge about cognitions (or metacognitions) can drive both appraisals and responses to negative cognitions leading to the selection, activation or interruption of strategies aimed at regulating them (Wells and Matthews 1994). Certain metacognitions appear to be detrimental in that they may activate ineffective self-regulatory strategies or favour their persistency. There is a broad agreement in distinguishing between two sub-type of problematic metacognitions, termed positive and negative metacognitions, and they can be applied to problematic use of online games (Spada and Caselli 2017; Marino and Spada 2017).

Positive metacognitions refer to the benefits of engaging in online gaming as a means of cognitive and affective regulation such as "online gaming will stop my worry" or "online gaming distracts my mind from my problems". Negative metacognitions concern the uncontrollability and dangers of thoughts about online gaming such as "once I start online gaming it is difficult to stop" or "thoughts about gaming interfere with my functioning". Positive metacognitions could play a central role in motivating individuals to engage in online gaming whilst negative metacognitions are thought to play a crucial role in the perpetuation of online gaming by becoming activated during and following engagement. Negative metacognitions may prevent any effective effort to stop gaming and/or trigger negative emotional states that compel a person to remain engaged, which in turn can increase the risk of developing a full-fledged addictive behaviour (Marino and Spada 2017; Spada et al. 2015).

In support of this view, metacognitions have been found to be associated with a wide array of psychological and behavioural problems (for a review, see Wells 2013), including the frequency and severity of a wide array of addictive behaviours (for a review see Spada et al. 2015; Hamonniere and Varescon 2018). These studies have led to the development and testing of specific metacognitive models of substance-related and addictive behaviours (Caselli et al. 2018a, b; Nikčević et al. 2017; Spada et al. 2013; Spada and Wells 2008). Within the area of Internet use, metacognitions have been found to be associated to levels of PIU (Spada et al. 2008; Spada and Marino 2017).

Spada and Caselli (2017) developed and validated the first self-report measure designed to assess metacognitions about online gaming and investigated the association between these metacognitions and both weekly online gaming hours and PIU controlling for negative affect (Spada and Caselli 2017). Findings from this preliminary study confirmed the distinction between positive and negative metacognitions about online gaming and revealed that metacognitions were significantly associated to both weekly online gaming hours and PIU over and above negative affect (Spada and Caselli 2017).

These preliminary findings suggest that metacognitions may play a role in the association between the frequency of online gaming and a broader pattern of PIU. 
On this basis, we put forward a metacognitive model of the relationship between negative affect, metacognitions about online gaming, frequency of online gaming and PIU as operationalized in Fig. 1. In this model, it is proposed that positive metacognitions about online gaming and negative affect lead to the engagement in online gaming as a means of negative affect regulation. Once gaming is initiated, it may bring to attentional absorption which is sustained by negative metacognitions about the uncontrollability of online gaming. These metacognitions prevent the activation of effective processes of monitoring and control online gaming activity thus favoring its continuation. Following a gaming episode, negative metacognitions about online gaming drive the appraisal of gaming activity and gaming-related thoughts as uncontrollable and dangerous that may lead to a further escalation of negative affect, which acts as a trigger of subsequent gaming episodes. The perseveration of online gaming through the flywheel of negative metacognitions may finally bring to PIU.

In this study we aimed to examine the relationships between negative affect, metacognitions about online gaming, weekly online gaming hours and PIU by testing the statistical fit of our metacognitive model in a community sample on online gamers. The hypothesised relationships among the variables were examined and the statistical fit of a path diagram was then tested (see Fig. 1).

\section{Method}

\section{Participants and Procedure}

Data was collected though an online questionnaire promoted by means of e-mail lists and advertisements (shared in social network groups and thematic forums). All participants were assured of the anonymity of their responses and they all agreed to give their consent to take part to the study. The Ethics Committee of Studi Cognitivi (Italy) gave formal approval for this research. Participants were recruited using an on-line snowballing sampling technique. They were aged between 18 and 56 years (mean age $=27$ years, $S D=5.65$ years; $93.3 \%$ males) with duration of online gaming use equal to 9.8 years $(S D=4.3)$. Those reporting to game less than $1 \mathrm{~h}$ per week $(n=2)$ and questionnaires including more than $20 \%$ of missing data in relevant scales $(n=59)$ were excluded. The remaining missing values $(n=2$ participants with 1 missing value) were imputed based on the participant's mean score on the relative scale (e.g., Marino et al. 2019). Therefore, the analyses were run on a final sample of 326 online gamers. They reported to play online for a mean of $18.50 \mathrm{~h}$ per week

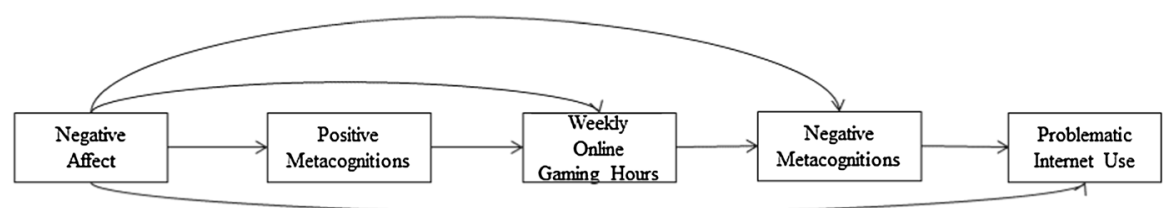

Fig. 1 Proposed theoretical model 
$(S D=19.52 \mathrm{~h})$, and the mean score on the Internet Addiction Test (IAT, Young $1998)$ was $42.58(S D=9.52$; range $=20-80)$.

\section{Measurement of Key Variables}

\section{Negative Affect}

The Hospital Anxiety and Depression Scale (HADS; Zigmond and Snaith 1983) was used to assess negative affect. This scale consists of 14 items, 7 assessing anxiety (e.g., "I feel tense or "wound up") and 7 assessing depression (e.g. "I feel as if I am slowed down"). Participants were asked to rate the extent to which they agreed with each item about of how they have been feeling over the past week on a 4-point scale (from (1) "not at all" to (4) "most of the time"). Items were summed to obtain a total score for negative affect. Higher scores represent higher levels of negative affect. The Italian version showed good psychometric properties (Costantini et al. 1999). The Cronbach's alpha for the scale in the present study was .85 (95\% CI .82-.87).

\section{Metacognitions}

The Metacognitions about Online Gaming Scale (MOGS; Spada and Caselli 2017) was used to assess positive and negative metacognitions about online gaming. The scale has two factors, each of which is assessed by 6 items: "positive metacognitions about online gaming" (P-MOG) referring to the usefulness of online gaming as cognitive-affective self-regulatory strategy (e.g., "Online gaming helps me to control my negative thoughts"); and "negative metacognitions about online gaming" (N-MOG) referring to the uncontrollability and dangers of online gaming and online gaming related thoughts (e.g., "I have no control over how much time I play"). Participants were asked to rate the extent of their agreement to each item on a 4-point scale (from (1) "do not agree" to (4) "agree very much"). Items were summed to obtain a score for both positive and negative metacognitions. Higher scores represent higher levels of metacognitions. The Cronbach's alpha for the positive and negative subscales in the present study were .84 (95\% CI .81-.87) and .86 (95\% CI .83-.88), respectively.

\section{Weekly Online Gaming Hours}

Participants were asked to indicate how many hours per week they usually spend gaming online.

\section{Problematic Internet Use}

The Internet Addiction Test (IAT; Young 1998) was used to assess the degree of problematic Internet use. The scale consists of 20 items (e.g., "How often do you choose to spend more time online over going out with others?"; "How often do you lose sleep due to late-night log-ins?"). Participants were asked to rate the frequency 
of each item on a 5-point scale (from (1) "never" to (5) "always"). Items were summed to obtain a total score for Internet addiction. Higher scores represent higher levels of PIU. The Italian version showed good psychometric properties (Faraci et al. 2013). The Cronbach's alpha for scale in the present study was .87 (95\% CI $.85-.89)$.

\section{Data Analyses}

Correlation analyses were conducted in order to test the associations between the variables of interest. The pattern of relationships specified by our theoretical model (Fig. 1) was examined through path analysis, using the package Lavaan (Rosseel 2012) of the software R (R Development Core Team 2013) and utilizing a single observed score for each construct included in the model. Specifically, the covariance matrix of the observed variable was analyzed with Maximum Likelihood method estimator and the Sobel test (also known as the product of coefficients approach; Baron and Kenny 1986; Hayes 2009) was used to test for mediation. To evaluate the goodness of fit of the model we considered the $\mathrm{R}^{2}$ of each endogenous variable and the total coefficient of determination (TCD; Bollen 1989; Jöreskog and Sörbom 1996). In the tested model, PIU was the dependent variable, negative affect was the independent variable, and weekly online gaming hours and positive and negative metacognitions about online gaming were the mediators (Fig. 1).

\section{Results}

Table 1 shows the means, standard deviations and bivariate correlations between the variables included in the study. As expected, all of the study variables were correlated with each other. In particular, a strong positive correlation was found between PIU and weekly online gaming hours spent gaming, and between negative affect and metacognitions about online gaming.

The theoretical model was tested including all the variables of interest. In this model, all path coefficients were significant at least at the $p<.01$ level. As shown in the Fig. 2, all the variables were interrelated with each other. Negative affect was directly associated with all other variables. Specifically, positive, strong and direct associations were found between negative affect and both positive and negative

Table 1 Means, standard deviations, and bivariate correlations for the study variables

\begin{tabular}{lrrllll}
\hline & M & SD & 1 & 2 & 3 & 4 \\
\hline 1. Weekly online gaming hours & 18.50 & 19.52 & 1 & & & \\
2. Problematic Internet use & 42.58 & 9.52 & $.39^{* *}$ & 1 & & \\
3. Positive metacognitions & 11.41 & 4.02 & $.24^{* *}$ & $.48^{* *}$ & 1 & \\
4. Negative metacognitions & 7.85 & 3.08 & $.36^{* *}$ & $.59^{* *}$ & $.25^{* *}$ & 1 \\
5. Negative affect & 22.68 & 2.48 & $.26^{* *}$ & $.56^{* *}$ & $.45^{* *}$ & $.49^{* *}$ \\
\hline
\end{tabular}

$* * p<.01 ; \mathrm{n}=326$ 


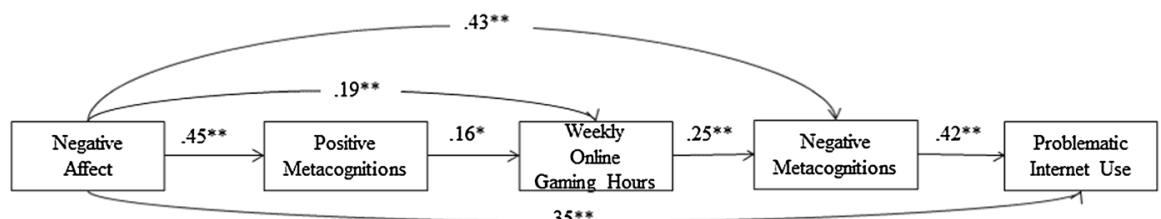

Fig. 2 Results of the path analytical model. Notes: ${ }^{*} p<.01,{ }^{* *} p<.001 ; \mathrm{n}=326$

metacognitions about online gaming. Moreover, negative metacognitions about online gaming were strongly linked to PIU.

Along with the direct paths, several indirect relationships were found significant at the 5\% level. Results of the Sobel test supported the mediating role of positive metacognitions about online gaming, weekly online gaming hours, and negative metacognitions about online gaming in the link between negative affect and PIU $(\beta=.01, p=.03)$. Specifically, negative affect has an indirect effect on weekly online gaming hours via positive metacognitions about online gaming $(\beta=.07, p=.01)$, and on negative metacognitions about online gaming via positive metacognitions online gaming and weekly online gaming hours $(\beta=.02, p=.02)$. Weekly online gaming hours also mediated the link between positive and negative metacognitions about online gaming $(\beta=.04, p=.02)$. The strongest indirect link was found between weekly online gaming hours and PIU via negative metacognitions about online gaming $(\beta=.11, p<.001)$.

The squared multiple correlations for the endogenous variables indicate that the model accounted for $44 \%$ of the variance for the outcome variable (PIU), and $30 \%$ of the variance for one mediator (negative metacognitions about online gaming) variable. Lower variance was observed for the other mediators (i.e. $20 \%$ for positive metacognitions about online gaming and $9 \%$ for weekly online gaming hours). Finally, the total amount of variance explained by the model (Total Coefficient of Determination, TCD $=.44$ ) indicated a good fit to the observed data. In terms of effect size, a TCD of .44 corresponds to a correlation of $r=.66$. According to the Cohen's (1988) traditional criteria, this is a large effect size.

\section{Discussion}

The aim of this study was to test the statistical fit of a metacognitive model of the interplay between negative affect, metacognitions about online gaming, weekly online gaming hours and PIU. Our metacognitive model was grounded in the metacognitive model of addictive behaviours (Spada et al. 2013, 2015). According to this formulation, addictive behaviours involve an inflexible perseveration of coping strategies that impair self-regulation and control because of the explicit or implicit impact of metacognitions.

In the hypothesized metacognitive model we proposed that positive metacognitions about online gaming lead online gaming as a means of emotional and cognitive self-regulation (e.g. reduce anxiety, suppress worries). Once online gaming 
is initiated, it can perseverate on the basis of negative metacognitions about online gaming that can be activated during and following engagement in online gaming. Negative metacognitions about online gaming relating to uncontrollability may prevent any effective effort to stop gaming while negative metacognitions about online gaming relating to the dangers of online gaming thoughts can trigger negative affective states that may compel an individual to remain engaged or return to engagement in order to regulate these states. The interruption of online gaming is gradually brought about by tiredness or external obstacles/distractions. These extreme and maladaptive stop signals hinder disconfirmation of metacognitive knowledge about uncontrollability on online gaming and indirectly prevent the adoption of effective self-regulation strategies. The perseveration of this self-feeding cycle may increase levels of PIU (Spada et al. 2015).

These findings have a number of possible implications for the assessment, conceptualisation, and treatment of PIU. In terms of assessment, information could be gathered not only in relation to the gaming activity per se but also, and perhaps mainly, to metacognitions about online gaming. The metacognitive model could also be used to guide the development of idiosyncratic case conceptualizations as well as socialize patients to the role of metacognitions in maladaptive self-regulation strategies and their consequences for symptoms, well-being, and quality of life. The present model can be illustrated to demonstrate the consistency in factors associated with the onset and perpetuation of online gaming and its impact of a more generalized PIU. The therapeutic implications may provide a foundation for the development of a Metacognitive Therapy for PIU and its multiple facet (like IGD) and can define specific targets for treatment potentially independent from the kind of online activity (King and Delfabbro 2018). These targets may refer to positive metacognitions about usefulness of online activity as a strategy for cognitive and affect self-regulation and negative metacognitions about uncontrollability of online activity. Such treatment should: (1) bring online activity (like gaming) under executive control; (2) modify positive and negative metacognitions; and (3) provide greater flexibility in mode of processing and self-regulation strategies that are not necessarily based on online activity. These goals may be achieved through the practice of attentional manipulation focused at improving adaptive goal-progress monitoring and the manipulation of stop signals. This should provide a means of amplifying self-attention thus increasing the flow of goal-progress information into processing (e.g. monitoring the ongoing impact of gaming on level of tension). Specific therapeutic strategies like metacognitive-delivered behavioural experiments and verbal reattribution techniques should aim to facilitate the abandonment of online gaming as a self-regulatory strategy and to modify positive and negative metacognitions about this process (Caselli et al. 2016, 2018).

The present results are preliminary in nature. The most important limitation is the absence of a longitudinal design that precludes causal test of the present model. Secondly, the community nature of the sample prevents conclusions at a clinical level. Thirdly, the use of snowballing recruitment techniques is subject to biased estimates. Fourthly, the presence of concurrent psychological disorder (which could account for the observed differences in outcome measures) was not assessed. However, controlling for anxiety and depression does provide a degree 
of confidence in the specificity of the results. Finally, online gaming specific measures (for example the Internet Gaming Disorder Scale; Monacis et al. 2016) could have been administered to provide an additional outcome measure to compare and contrast with weekly online gaming hours. Future research will need to test the proposed model with problematic online gaming as an outcome, use of longitudinal designs, and include clinical populations.

Despite the above limitations, we believe the present findings might provide a step forward in a cognitive conceptualization of the maintenance of problematic online gaming that integrates metacognitive knowledge with self-regulation processes.

Open Access This article is licensed under a Creative Commons Attribution 4.0 International License, which permits use, sharing, adaptation, distribution and reproduction in any medium or format, as long as you give appropriate credit to the original author(s) and the source, provide a link to the Creative Commons licence, and indicate if changes were made. The images or other third party material in this article are included in the article's Creative Commons licence, unless indicated otherwise in a credit line to the material. If material is not included in the article's Creative Commons licence and your intended use is not permitted by statutory regulation or exceeds the permitted use, you will need to obtain permission directly from the copyright holder. To view a copy of this licence, visit http://creativecommons.org/licen ses/by/4.0/.

\section{References}

American Psychiatric Association. (2013). Diagnostic and statistical manual of mental disorders (5th ed). American Psychiatric Association.

Baron, R. M., \& Kenny, D. A. (1986). Moderator-mediator variables distinction in social psychological research: Conceptual, strategic, and statistical considerations. Journal of Personality and Social Psychology, 51, 1173-1182.

Beard, K. W., \& Wolf, E. M. (2001). Modification in the proposed diagnostic criteria for Internet addiction. CyberPsychology and Behavior, 4, 377-383.

Billieux, J., Schimmenti, A., Khazaal, Y., Maurage, P., \& Heeren, A. (2015). Are we overpathologizing everyday life? A tenable blueprint for behavioral addiction research. Journal of Behavioral Addictions, 4, 119-123.

Bollen, K. A. (1989). Structural equations with latent variables. New York: Wiley.

Caselli, G., Fernie, B., Canfora, F., Mascolo, C., Ferrari, A., Antonioni, M., et al. (2018a). The metacognitions about gambling questionnaire: Development and psychometric properties. Psychiatry Research, 261, 367-374.

Caselli, G., Gemelli, A., Spada, M. M., \& Wells, A. (2016). Experimental modification of perspective on thoughts and metacognitive beliefs in alcohol use disorder. Psychiatry Research, 244, 57-61.

Caselli, G., Martino, F., Spada, M. M., \& Wells, A. (2018b). Metacognitive therapy for alcohol use disorder: A systematic case series. Frontiers in Psychology, 9(2619), 1-11.

Cohen, J. (1988). Statistical power analysis for behavioral science (2nd ed.). Hillsdale: Erlbaum.

Cole, H., \& Griffiths, M. D. (2007). Social interactions in massively multiplayer online role-playing gamers. Cyberpsychology and Behavior, 10, 575-583.

Costantini, M., Musso, M., Viterbori, P., Bonci, F., Del Mastro, L., Garrone, O., et al. (1999). Detecting psychological distress in cancer patients: Validity of the Italian version of the hospital anxiety and depression scale. Supportive Care in Cancer, 7, 121-127.

Faraci, P., Craparo, G., Messina, R., \& Severino, S. (2013). Internet Addiction Test (IAT): Which is the best factorial solution? Journal of Medical Internet Research, 15, e225.

Hamonniere, T., \& Varescon, I. (2018). Metacognitive beliefs in addictive behaviours: A systematic review. Addictive Behaviors, 85, 51-63. 
Hayes, A. F. (2009). Beyond Baron and Kenny: Statistical mediation analysis in the new millennium. Communication Monographs, 76, 408-420.

Jöreskog, K. G., \& Sörbom, D. (1996). LISREL 8: User's reference guide. Chicago: Scientific Software International.

King, D., \& Delfabbro, P. (2018). Internet gaming disorder: Theory, assessment, treatment, and prevention. Cambridge: Academic Press.

Laconi, S., Kaliszewska-Czeremska, K., Gnisci, A., Sergi, I., Barke, A., Jeromin, F., et al. (2018). Crosscultural study of problematic Internet use in nine European countries. Computers in Human Behavior, 84, 430-440.

Marino, C., Marci, T., Ferrante, L., Altoè, G., Vieno, A., Simonelli, A., et al. (2019). Attachment and problematic Facebook use in adolescents: The mediating role of metacognitions. Journal of Behavioral Addictions, 8, 63-78.

Marino, C., \& Spada, M. M. (2017). Dysfunctional cognitions in online gaming and internet gaming disorder: A narrative review and new classification. Current Addiction Reports, 4, 308-316.

Monacis, L., Palo, V. D., Griffiths, M. D., \& Sinatra, M. (2016). Validation of the internet gaming disorder scale-Short-form (IGDS9-SF) in an Italian-speaking sample. Journal of Behavioral Addictions, 5, 683-690.

Moreno, M. A., Eickhoff, J., Zhao, Q., Young, H. N., \& Cox, E. D. (2019). Problematic Internet use: A longitudinal study evaluating prevalence and predictors. The Journal of Pediatrics, 1, 100006.

$\mathrm{Ng}$, B. D., \& Wiemer-Hastings, P. (2005). Addiction to the internet and online gaming. Cyberpsychology and Behavior, 8, 110-113.

Nikčević, A. V., Alma, L., Marino, C., Kolubinski, D., Ylmaz-Samanci, A. E., Caselli, G., et al. (2017). Modelling the contribution of negative affect, outcome expectancies and metacognitions to cigarette use and nicotine dependence. Addictive Behaviors, 74, 82-89.

Peters, C. S., \& Malesky, L. A. (2008). Problematic usage among highly-engaged players of massively multiplayer online role playing games. Cyberpsychology and Behavior, 11, 480-483.

R Core Team. (2013). R: A language and environment for statistical computing [Computer software manual]. Vienna, Austria. Available from http://www.R-project.org/.

Rosseel, Y. (2012). Lavaan: An R package for structural equation modeling. Journal of Statistical Software, 48, 1-36.

Spada, M. M. (2014). An overview of problematic Internet use. Addictive Behaviors, 39, 3-6.

Spada, M. M., \& Caselli, G. (2017). The metacognitions about online gaming scale: Development and psychometric properties. Addictive Behaviors, 64, 281-286.

Spada, M. M., Caselli, G., Nikčević, A. V., \& Wells, A. (2015). Metacognition in addictive behaviors. Addictive Behaviors, 44, 9-15.

Spada, M. M., Caselli, G., \& Wells, A. (2013). A triphasic metacognitive formulation of problem drinking. Clinical Psychology and Psychotherapy, 20, 494-500.

Spada, M. M., Langston, B., Nikčević, A. V., \& Moneta, G. B. (2008). The role of metacognitions in problematic Internet use. Computers in Human Behavior, 24, 2325-2335.

Spada, M. M., \& Marino, C. (2017). Metacognitions and emotional regulation as predictors of problematic Internet use in adolescents. Clinical Neuropsychiatry, 14, 59-63.

Spada, M. M., \& Wells, A. (2008). Metacognitive beliefs about alcohol use: Development and validation of two self-report scales. Addictive Behaviors, 33, 515-527.

Van Rooij, A. J., Schoenmakers, T. M., Van de Eijnden, R. J., \& Van de Mheen, D. (2010). Compulsive Internet use: The role of online gaming and other internet applications. Journal of Adolescent Health, 47, 51-57.

Wells, A. (2013). Advances in metacognitive therapy. International Journal of Cognitive Therapy, 6, 186-201.

Wells, A., \& Matthews, G. (1994). Attention and emotion: A clinical perspective. Hove: Lawrence Erlbaum Associates.

Wells, A., \& Matthews, G. (1996). Modelling cognition in emotional disorder: The S-REF model. Behaviour Research and Therapy, 34, 881-888.

World Health Organization. (2018). International classification of diseases for mortality and morbidity statistics (11th Revision). Retrieved from https://icd.who.int/browse11/1-m/en.

Young, K. S. (1998). Internet addiction: The emergence of a new clinical disorder. Cyberpsychology and Behavior, 1, 237-244.

Zigmond, A. S., \& Snaith, R. P. (1983). The hospital anxiety and depression scale. Acta Psychiatrica Scandinavica, 67, 361-370. 
Publisher's Note Springer Nature remains neutral with regard to jurisdictional claims in published maps and institutional affiliations. 